\title{
Internationally trained medical physicists should be certified, through ACPSEM, in order to be employed in Australia and New Zealand as Radiation Oncology Medical Physicists
}

\author{
Jackson M. Zifodya ${ }^{1} \cdot$ Michael Barnes $^{2} \cdot$ Clive Baldock $^{3}$ \\ Accepted: 23 December 2021 / Published online: 30 January 2022 \\ (c) Australasian College of Physical Scientists and Engineers in Medicine 2021
}

\section{Introduction and overview: Clive Baldock, moderator}

In Australia and New Zealand, the Australasian College of Physical Scientists and Engineers in Medicine (ACPSEM) is responsible for the training of graduate physical scientists for Certification as Medical Physics Specialists and Radiopharmaceutical Scientists [1]. This training is administered through the Training Education and Assessment Program (TEAP) which combines post-graduate study with full-time competency-based training and assessment in an ACPSEM accredited clinical department. In radiation oncology medical physics, diagnostic imaging medical physics, and radiopharmaceutical science, TEAP is the only pathway to certification.

The certification pathway applies only to recent university graduates. Experienced radiation oncology and diagnostic imaging medical physics practitioners seeking an ACPSEM qualification bypass the certification process and are required only to apply for registration as a Qualified Medical Physics Specialist under the Experienced Candidate category with certification awarded by the ACPSEM Professional Standards Board. Newly certified medical physics specialists and radiopharmaceutical scientists are automatically admitted to the ACPSEM Register of Qualified Medical Physics Specialists and Radiopharmaceutical Scientists.

The three ACPSEM training and education programs ensure the safety and efficacy of the medical physics and

Clive Baldock

C.Baldock@westernsydney.edu.au

1 North West Cancer Centre, Tamworth Hospital, Dean Street, Tamworth, NSW 2340, Australia

2 Calvary Mater Newcastle Public Hospital, Edith Street, Waratah, NSW 2298, Australia

3 Graduate Research School, Western Sydney University, Penrith, NSW 2747, Australia radiopharmaceutical science professions in Australia and New Zealand, and to the professional standards pertinent to their practice with participants educated and trained consistent with established standards of expertise, safe practice, quality assurance, and regulatory compliance.

In this topical debate, Jackson Zifodya and Michael Barnes debate whether internationally trained medical physicists should be certified, through ACPSEM, in order to work in Australia and New Zealand as Radiation Oncology Medical Physicists (ROMPs). ${ }^{1}$

Arguing for the proposition is Jackson Zifodya. Jackson received his MSc in Medical Physics in 1999, from the University of Surrey, UK. He attained his certificate as a Chartered Scientist, from the Science Council, Institute of Physics and Engineering in Medicine, UK, in 2005. His medical physics career spans three continents. He started as a Junior Physicist in 1995 at Parirenyatwa Hospital, in Zimbabwe. He then moved to the UK, in 2000, and worked for Maidstone Hospital, Maidstone, St Bartholomew's Hospital, London and Derriford Hospital, Plymouth. In the UK his roles included Head of Dosimetry and Consultant Physicist. He then moved to Tamworth, Australia, in 2013, where, as the Director of Medical Physics, he set up a new radiotherapy department, the North West Cancer Centre. In his time, he has commissioned more than 10 linear accelerators. His current interests include automating clinical processes, external beam treatment planning and radiation detectors.

\footnotetext{
${ }^{1}$ Contributors to Topical Debates are selected for their knowledge and expertise. Their position for or against a proposition may or may not reflect their personal opinions.
} 


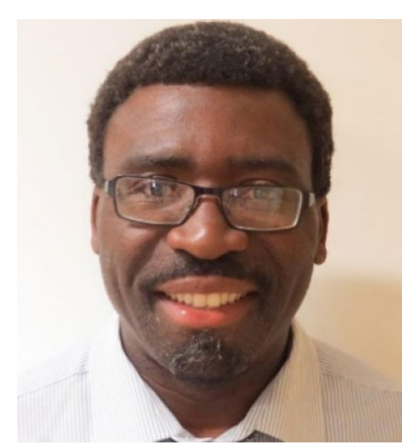

Jackson Zifodya

Arguing against the proposition is Michael Barnes, a Senior Medical Physics Specialist who has worked at the Calvary Mater Newcastle $(\mathrm{CMN})$ since 2004. Michael was the inaugural CMN registrar from the first NSW TEAP intake in 2004 and was awarded a Masters by Research in 2009 from the University of Newcastle. Michael opted to complete certification via the ACPSEM Accreditation in Radiotherapy Equipment Commissioning and Quality Assurance (ARECQA) program in 2012 and is currently enrolled in a $\mathrm{PhD}$. Michael has successfully trained and supervised TEAP registrars and specialises in linear accelerator commissioning, quality assurance and dosimetry and in this capacity has trained numerous TEAP registrars in Modules 3 and 4 of the TEAP Clinical Training Guide.

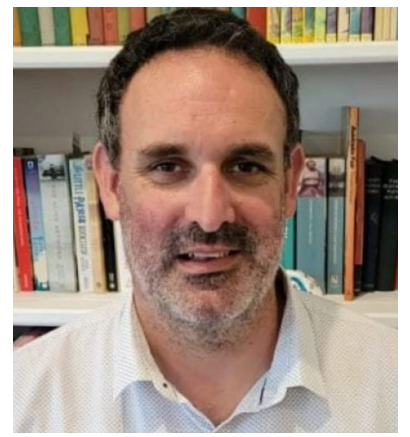

Michael Barnes

\section{Opening statement-Jackson Zifodya}

According to the Cambridge Dictionary, certification is "the process of earning an official document, or the act of providing an official document, as proof that something has happened or been done" or "a document proving that someone is qualified for a particular job, or that something is of good quality" [2]. Wikipedia says "Certification is the formal attestation or confirmation of certain characteristics of an object, person, or organization. This confirmation is often, but not always, provided by some form of external review, education, assessment, or audit" [3]. The current Australian system for the certification of ROMPs follows the Wikipedia definition where confirmation of qualifications/suitability are assessed through a fairly rigorous process.

If the purpose of certification is to determine if an individual is fit to practice as a ROMP in Australia or New Zealand, then the current system of employing internationally trained Physicists makes a mockery of the ACPSEM Certification ideology. Currently certification is done through ACPSEM. Employment of ROMPs is, however, at the discretion of the Australia's Local Health Districts (LHD) and New Zealand's District Health Boards (DHB). As an employer, the LHD/DHB can appoint a ROMP who has no ACPSEM certification, and they can work in the LHD/DHB for as long as their services are required. The only difference, in the case of Australia, is the slightly lower remuneration for the non-certified ROMPs when compared to certified colleagues on the same pay band.

Following the Cambridge Dictionary's definition [2], all that is required is to simply present a certificate from the international organisation where you trained. Well, not so simple. How would we ensure we employ the most adept candidates? The answer to how we assess whether an internationally trained ROMP is "fit for purpose" is in my opinion already there. There is no doubt that some form of assessment is required. The most logical way is to incorporate certification into the initial employment interview by the LHD/DHB. The interview would have to be tailored to include aspects that ascertain the individual satisfies the LHD/DHB's requirements as well as ACPSEM's certification standards. The adaptation could include requesting certification documents from the country of domicile as well as having a College appointed ACPSEM certified ROMP on the interview panel. The ACPSEM could also have a list of training 'dimensions' they deem essential that could be used at interview for assessment. This list could incorporate international benchmarks for assessing qualifications for the purposes of certification [4]. For purposes of employment, Heads of Medical Physics Departments in New Zealand consider HCPC/IPEM (UK) [5], ABR/ABMP (USA) [6], CCPM (Canada) [7], DGMP (Germany) [8] certification as broadly similar to the standards set by ACPSEM [9]. There is no compelling reason why an arrangement similar to this cannot be formalised by the ACPSEM without the need for reassessment.

It cannot be discounted that an international candidate could be coming from a system that has a better training program than Australia has and therefore that training program potentially produces equally competent, if not better, physicists. Some could have PhDs in novel research areas 
that have significantly advanced radiotherapy, and improved patient outcomes, as well as having well developed research skills of benefit to the profession [10]. What then is the purpose of undergoing a tortuous independent process, well after being employed, so an individual can be classed as certified? They are already doing the job if the LHD/DHB has employed them.

\section{Opening statement-Michael Barnes}

The purpose of certification is to attest that an individual has attained a minimum standard of competency. Hence, this proposition is a question of assessment rather than training. Certification helps to achieve a homogeneous professional standard which ensures quality and safety in radiation medicine [11]. As such, certification itself is not in question here. The question is specific to international ROMPs who wish to practice in Australia and New Zealand and whether their international qualifications should be recognized as fulfilling the local minimum standard and they therefore should be considered certified and allowed to practice locally.

It is desirable to provide a pathway for demonstrated high performing foreign ROMPs to practice locally. However, in the interest of maintaining standards, the question is how to identify these individuals from those overseas applicants who need further training and assessment before being considered to meet the local minimum standard. As I see it, there are two ways in which this can realistically be addressed.

One solution is to have a recognition system whereby certain overseas certifications are deemed as acceptable to allow a graduate to practice locally. In such a recognition system, individuals are not assessed, but foreign certification programs are. The difficulty with this is that there is no local control over foreign programs and only limited control exists in an international certification to which there has been local input and potential endorsement [12]. This brings a whole host of practical problems regarding determination and maintenance of equivalence, standard, and consistency and this makes it difficult to assure that each individual meets the local minimum standard, especially over time as certification programs and local minimum standards evolve.

The alternative is for all overseas applicants to follow a local assessment process similar to local ROMPs regardless of what prior certification they may have. This ensures equality in the assessment process between local and international applicants leading to consistent minimum competency levels across the local profession. Additionally, it provides security for employers and regulatory authorities that all practicing individuals are indeed qualified to perform the duties required of them.
Barring a further alternative, the question comes down to whether an overseas certification, with the associated lack of control and difficulties in assuring consistent equivalence, is recognized or whether each applicant is individually assessed with the associated burden this places on individuals which may dissuade some high quality applicants from seeking to practice in Australia and New Zealand. While not perfect, I'd suggest that the latter is the superior solution.

\section{Rebuttal—Jackson Zifodya}

It is worth reiterating that employment of ROMPs into active medical physics roles, in Australia or New Zealand, is not undertaken by ACPSEM but rather by the LHD/DHB. Staffing, retention and recruitment of qualified ROMPs is an ongoing issue which is well documented [13-16]. The Tripartite National Strategic Plan for Radiation Oncology, Stakeholder Consultation, included an increasing dependence on overseas recruitment as one of the factors that impact on the radiation oncology workforce [17]. The Australian Government's Department of Health also cited recruiting ROMPs from overseas as a targeted recruitment strategy [18]. The current system of certifying overseas trained ROMPs puts paid to these strategies.

The overseas trained ROMPs often perform everything an ACPSEM certified ROMP would do. At the end of all this, patients are treated in these departments without much fuss. To date there have been no reported incidence where a noncertified ROMP has caused a major incident [19]. This just goes to show that the current system of employing ROMPs is adequate in weeding out the undesirables and as such only needs tweaking to make it more robust which would include involving ACPSEM at the interview stage and subsequent certification.

All employed ROMPs owe their current positions to the interview process, including my esteemed colleague. Yes, the process might have shortcomings [20], hence why it should be reviewed to include the checks mentioned before. We should embrace the International Medical Physics Certification Board (IMPCB) which is working to develop an international certification system endorsed by several national Medical Physics Societies [21]. Currently, certification by IMPCB is not enough as one still has to undergo the ACPSEM certification process.

In conclusion, my argument is not against certification but rather the current certification process for overseas trained ROMPs. The process in place is akin to employing a chef (employed through interviews) to cook for the Royal family and then 6 months down the line asking that chef if they know what an onion or a tomato is. It beggars belief as to how this can be viewed as logical. Closing the stable door after the horse has bolted could have dire consequences. 
Certification should be done at the earliest stage of the employment process and therefore should be incorporated into the interview stage.

\section{Rebuttal—Michael Barnes}

I dispute any assertion by my esteemed colleague that the job interview process performed at the local department, even modified, is superior to the certification process for assuring individual competency. Certification needs to be standardized across Australia and New Zealand regardless of an individual's prior qualifications or where they were trained to help ensure a consistent minimum standard of practice across Australia and New Zealand.

The interview process has been considered to be inaccurate for assessing applicant competency [22]. However, my colleague isn't suggesting that the interview process as performed currently is sufficient but that the process could be both improved to better assess applicant competency and that the local department, potentially assisted by the ACPSEM, is the appropriate body for assessing suitability of an applicant's qualifications.

In the absence of certification, modifying the interview process to allow better demonstration of competency would improve applicant assessment. However, when improvements to the interview process, such as those put forward by my colleague, are extrapolated towards what is optimal for assuring a minimum standard of practice then one arrives at the certification process. This is due to the advantages of having expert assessors, consistency of assessment and removal of the burden off local departments. Having an ACPSEM appointed representative at all interviews is impractical and potentially costly and without the reference point of a common certification, inconsistencies will arise. While better than nothing, such a system would prove less consistent and therefore potentially lead to inappropriate local professional standards compared to a common, standardized certification process developed specifically for the local jurisdiction. Current international benchmarks for recognition of equivalence are flawed as presented in my opening statement.

The interview process is important for assessing an applicant's experience level, areas of interest, and non-technical attributes. However, a certification process is best for assessing technical competency and this needs to be standardized across a jurisdiction to ensure consistency. Hence, both, an interview and a certification specific to Australia and New Zealand are required for each and every individual to assure a safe and competent local ROMP workforce. As such international physicists should be required to pass certification in Australia and New Zealand to be allowed to practice as ROMPs.

\section{References}

1. Australasian College of Physical Scientists and Engineers in Medicine (2019) Certification. https://www.acpsem.org.au/Our-Work/ TEAP-Certification. Accessed 1 Dec 2021

2. Certification (2021) Cambridge Dictionary. https://dictionary. cambridge.org/dictionary/english/certification. Accessed 1 Nov 2021

3. Wikipedia (2021) Certification. https://en.wikipedia.org/wiki/ Certification. Accessed 31 May 2021

4. International Atomic Energy Agency (2021) Accreditation and Certification. https://humanhealth.iaea.org/HHW/MedicalPhysics/ TheMedicalPhysicist/EducationandTrainingRequirements/Accre ditation_and_Certification/index.html. Accessed 6 Aug 2021

5. IPEM (2021) Regulation and Registration. https://www.ipem.ac. uk/trainingworkforce/regulationandregistration.aspx. Accessed 9 Aug 2021

6. American Board of Radiology (2021) Initial Certification. https:// www.theabr.org/medical-physics/initial-certification. Accessed 9 Aug 2021

7. CCPM (2021) Eligibility. https://ccpm.ca/ccpm-english/main/certi fication/eligibility.html Accessed Aug 92021

8. DGMP (2021) Fachkunde \& Fachanerkennung. https://www.jmp. dgmp.de/de-DE/686/fachkunde-fachanerkennung/ Accessed Aug 92021

9. New Zealand Medical Physicist Heads of Department (June 2013). Agreement on professional standards for medical physicists in New Zealand. Unpublished.

10. Booth J, Whittaker M, Baldock C (2020) In the future all accredited radiotherapy physicists should have a PhD. Phys Eng Sci Med 43:1139-1143

11. International Atomic Energy Agency (2013) Roles and responsibilities, and education and training requirements for clinically qualified medical physicists. IAEA Human Health Series, no. 25. https://www-pub.iaea.org/MTCD/Publications/PDF/Pub1610_ web.pdf. Accessed Nov 12021

12. International Medical Physics Certification Board (2021). https:// www.impcbdb.org. Accessed Jul 292021

13. Round WH (2013) A 2012 survey of the Australasian clinical medical physics and biomedical engineering workforce. Austral Phys Eng Sci Med 36:147-157

14. Humphreys J, Wakerman J, Kuipers P et al (2017) Improving workforce retention: developing an integrated logic model to maximise sustainability of small rural and remote health care services. https://rsph.anu.edu.au/files/full_report_10797.pdf. Accessed Nov 12021

15. Schofield D, Callander E, Kimman M (2012) Projecting the radiation oncology workforce in Australia. Asian Pac J Cancer Prev 13:1159-1166

16. Dempsey C (2018) Medical physics workforce modelling: do we need what we want? Austral Phys Eng Sci Med 41:565-566

17. Radiation Oncology Tripartite Committee (2012) Planning for the best. http://www.radiationoncology.com.au/supporting-docs/Plann ingForTheBest-TripartiteNationalStrategicPlanForRadiationOn cology.2012-2022.pdf. Accessed Nov 12021

18. Department of Health (2011) Appendix 1-Summary of state and territory workforce initiatives outlined in the HealthConsult Report. https://www1.health.gov.au/internet/publications/publi shing.nsf/Content/RORICworkforcereformframework-lp RORIC workforcereformframework-appendix 1. Accessed Sep 202021

19. Knöös T (2017) Lessons learnt from past incidents and accidents in radiation oncology. Clin Oncol 29:557-561

20. Maharjan P (2018) Strengths and limitations of interview. https:// www.businesstopia.net/human-resource/strengths-and-limit ations-interview Accessed Jul 282021 
21. International Organization for Medical Physics (2021) IOMP history. https://www.iomp.org/iomp-history/. Accessed Sep 212021

Publisher's Note Springer Nature remains neutral with regard to jurisdictional claims in published maps and institutional affiliations.

22. Friedman R (2014) The best place to work: the art and science of creating an extraordinary workplace. Perigee, Penguin Group, New York 